\title{
Five cases of cyclical Cushing's syndrome
}

\author{
A BREW ATKINSON, A LAURENCE KENNEDY, DENNIS J CARSON, DAVID R HADDEN, \\ JOHN A WEAVER, BRIAN SHERIDAN
}

\begin{abstract}
Reported cases of cyclical Cushing's syndrome are rare. Of 14 successive patients with Cushing's syndrome nine collected sequential urine samples for the estimation of cortisol:creatinine ratio. Five had cyclical Cushing's syndrome while two had considerable variation in urinary cortisol excretion without a cyclical pattern being established. Two of the five patients with a cyclical syndrome had paradoxical responses to dexamethasone. In only one patient with a cyclical pattern did the cortisol: creatinine ratio fall after treatment with bromocriptine or cyproheptadine, or both.

The high incidence of the cyclical form of Cushing's syndrome has important clinical implications. A high index of suspicion of the syndrome is required in patients with symptoms or signs of Cushing's syndrome but with normal cortisol values, in patients with fluctuating cortisol values, and in patients with anomalous responses to dexamethasone. Because of possible variations in steroidogenesis the results of drug studies in Cushing's syndrome must be interpreted cautiously.
\end{abstract}

\section{Introduction}

In 1971 Bailey described a patient with Cushing's syndrome due to a bronchial adenoma in whom there appeared to be cycles of excess cortisol secretion.' Since then a few more cases have been de-

Sir George E Clark Metabolic Unit, Royal Victoria Hospital, Belfast BT12 6BA

A BREW ATKINSON, MD, MRCP, consultant physician

A LAURENCE KENNEDY, MD, MRCP, consultant physician

DAVID R HADDEN, MD, FRCP, consultant physician

JOHN A WEAVER, MD, FRCP, consultant physician

Royal Belfast Hospital for Sick Children and Departmeni of Child Health, Queen's University, Belfast

DENNIS J CARSON, MB, MRCP, consultant lecturer

Regional Endocrine Laboratory, Royal Victoria Hospital, Belfast BT12 6BA BRIAN SHERIDAN, MSC, top grade biochemist

Correspondence to: Dr Atkinson. scribed $^{2-9}$ in which regular cycles of excess cortisol production (varying from 12 hours to 85 days) occurred, though in only a few of these cases did cortisol production fall in the troughs to normal values. Cyclical Cushing's syndrome is diagnosed by showing three peaks and two troughs of cortisol production with similar distances between the peaks.

In virtually all patients with Cushing's syndrome cortisol production is considered to exceed normal at all times.' Exceptions have, however, been described and ascribed to $(a)$ random fluctuations in cortisol secretion, (b) spontaneous remissions of the Cushingoid state, and (c) cyclical patterns of cortisol secretion. Patients with anomalous responses to tests of the hypothalamic-pituitary-adrenal axis, particularly so called paradoxical responses to dexamethasone, have also been described. ${ }^{10}$ In the latter steroid outputs rise rather than fall during dexamethasone administration. The reason for the paradoxical responses has remained ill defined, but Brown et al suggested that they may be due to variable secretion of cortisol rather than to a true effect of dexamethasone on cortisol production. ${ }^{2}$

Over the past two years we have seen 14 patients with Cushing's syndrome, nine of whom have provided sequential daily urine samples enabling us to show a cyclical pattern of Cushing's syndrome in five of them. The studies performed to establish cyclical patterns, the response of these patients to bromocriptine and cyproheptadine, and the implications of our studies for the diagnosis of patients with Cushing's syndrome are described here.

\section{Patients and methods}

None of the 14 patients drank alcohol to excess. The first patient (case 1) had anomalous responses to low and high dose dexamethasone and was shown to have a cyclical form of Cushing's syndrome secondary to a basophil adenoma of the pituitary (see below). Eight of the remaining 13 patients provided sequential daily urine samples for at least 27 days; the four who were shown to have cyclical Cushing's syndrome (cases 2-5) are also described below. In another two there was considerable variation in the urinary cortisol excretion but a cyclical pattern was not established. Because of their more advanced clinical state we could not study the five other patients in such detail.

Serum samples were stored at $-20^{\circ} \mathrm{C}$ before cortisol concentrations were estimated by a direct radioimmunoassay featuring an iodine -125 radioligand and a solid phase antibody. ${ }^{11}$ For estimating urinary free cortisol the 
urine was extracted with dichloromethane and the assay for free cortisol performed on the dried solvent residue. ${ }^{11}$ The upper limit of normal was 450 $\mathrm{nmol} / 24 \mathrm{~h}(163 \mu \mathrm{g} / 24 \mathrm{~h})$. Urinary creatinine was determined by autoanalyser using the Jaffe reaction. ${ }^{12}$ The urinary free cortisol:creatinine ratio was calculated by dividing the urinary free cortisol value in $\mathrm{nmol} / 1$ by the urinary creatinine value in $\mathrm{mmol} / 1$. An aliquot of either a 24 hour collection of urine or the first specimen of urine passed on rising was estimated after storage at $-20^{\circ} \mathrm{C}$.

The 24 hour urinary free cortisol:creatinine ratio was compared with the early morning ratio on 46 samples from 46 patients and there was a microscopy had ultrastructural features in keeping with a corticotroph adenoma. Prolonged clinical remission (117 days) has been achieved by transsphenoidal surgery.

CASE 2

A 36 year old woman presented with facial swelling, hirsutism, striae, and weight gain. Twenty one years earlier she had had rapid weight gain and amenorrhoea for three years and had been investigated for Cushing's

Results of urinary free cortisol excretion ( $n$ mol/24 h) during low and high dose dexamethasone suppression tests on five patients with cyclical Cushing's syndrome. Only in case 1 was there a basal collection between the low and high dose tests

\begin{tabular}{|c|c|c|c|c|c|c|c|c|c|}
\hline \multirow[b]{2}{*}{ Drug } & \multirow{2}{*}{$\frac{\text { Case } 1}{\text { Test } 1}$} & \multicolumn{2}{|c|}{ Case 2} & \multicolumn{2}{|c|}{ Case 3} & \multicolumn{2}{|c|}{ Case 4} & \multicolumn{2}{|c|}{ Case 5} \\
\hline & & Test 1 & Test 2 & Test 1 & Test 2 & Test 1 & Test 2 & Test 1 & Test 2 \\
\hline Basal value & 131 & 766 & 2091 & 690 & 678 & 195 & 61 & 688 & 981 \\
\hline Basal value & 163 & 1600 & 3526 & 730 & 1483 & 245 & 73 & 655 & 923 \\
\hline Dexamethasone & & & & & & & & & \\
\hline $\begin{array}{l}0.5 \mathrm{mg} \text { four times a day } \\
\text { Dexamethasone }\end{array}$ & 2675 & 1489 & 1992 & 343 & 591 & 585 & 138 & 336 & 310 \\
\hline $0.5 \mathrm{mg}$ four times a day & 4039 & 2052 & 2280 & 270 & 167 & 1193 & 216 & 240 & 261 \\
\hline Basal value & 690 & & & & & & & & \\
\hline Basal value & 103 & & & & & & & & \\
\hline Dexamethasone & & & & & & & & & \\
\hline $2 \mathrm{mg}$ four times a day & 3776 & 1099 & & & & 3390 & 459 & 118 & 139 \\
\hline Dexamethasone & & & & & & & & & \\
\hline $2 \mathrm{mg}$ four times a day & 4320 & 1091 & & & & 6100 & & 64 & 45 \\
\hline
\end{tabular}

correlation of $0 \cdot 92$. Our upper limit of normal for cortisol:creatinine ratio in an early morning specimen of urine was 50 . There were no differences in urinary free cortisol values among fresh samples stored at room temperature for intervals of up to seven days before storage and subsequent analysis. This experiment was performed to ensure that mailing urine specimens did not lead to spurious results.

Blood was withdrawn into tubes containing edetic acid. The samples were centrifuged promptly and the plasma stored at $-20^{\circ} \mathrm{C}$ until estimation of plasma adrenocorticotrophic hormone by radioimmunoassay, using reagents supplied by Immuno Nuclear Corporation. The normal range was $<75 \mathrm{ng} / 1$. The between batch coefficient of variation was $7 \cdot 1 \%$ for a sample with a mean value of $98 \mathrm{ng} / 1$.

\section{Case reports}

CASE 1

A 71 year old woman was referred to us with a biochemically confirmed diagnosis of Cushing's syndrome. Six months earlier she had noticed deepening of skin colour associated with extreme tiredness. She was hypertensive. Low dose and high dose dexamethasone suppression tests were performed (see table). During the basal period the serum and urinary free cortisol concentrations were normal but became raised during low dose dexamethasone administration. Afterwards they fell to normal but again rose during high dose dexamethasone administration.

The patient's urinary free cortisol secretion and serum cortisol were then observed for 22 days (fig 1). The serum and urinary cortisol peaks and nadirs correlated closely with plasma adrenocorticotrophic hormone values, providing evidence for dependence on adrenocorticotrophic hormone. The patient's tiredness and facial plethora were greater when the urinary cortisol values were abnormal. Blood pressure showed no cyclical variation. There were two distinct rhythms in cortisol production. One rhythm consisted of a period of 40 days of excess cortisol production followed by 60-70 days of normal production (fig 2). During the period of observation in hospital both the urinary cortisol:creatinine ratio and the urinary free cortisol showed cyclical variation, indicating that the cortisol:creatinine ratio was a valid reflection of cortisol secretion. Within the period of excess cortisol production there was a second rhythm consisting of peaks of cortisol production every three to six days with troughs of normal cortisol production. Since this patient differed from the other four in this respect and since these rhythms made interpretation of drug studies difficult she has been described in detail elsewhere. ${ }^{13}$

Neither bromocriptine nor cyproheptadine had an effect on cortisol production. A coronal plane computed tomogram showed a $7 \mathrm{~mm}$ pituitary microadenoma. Computed tomography of the lungs was normal. A pituitary surgery a basophil adenoma was removed, which on electron

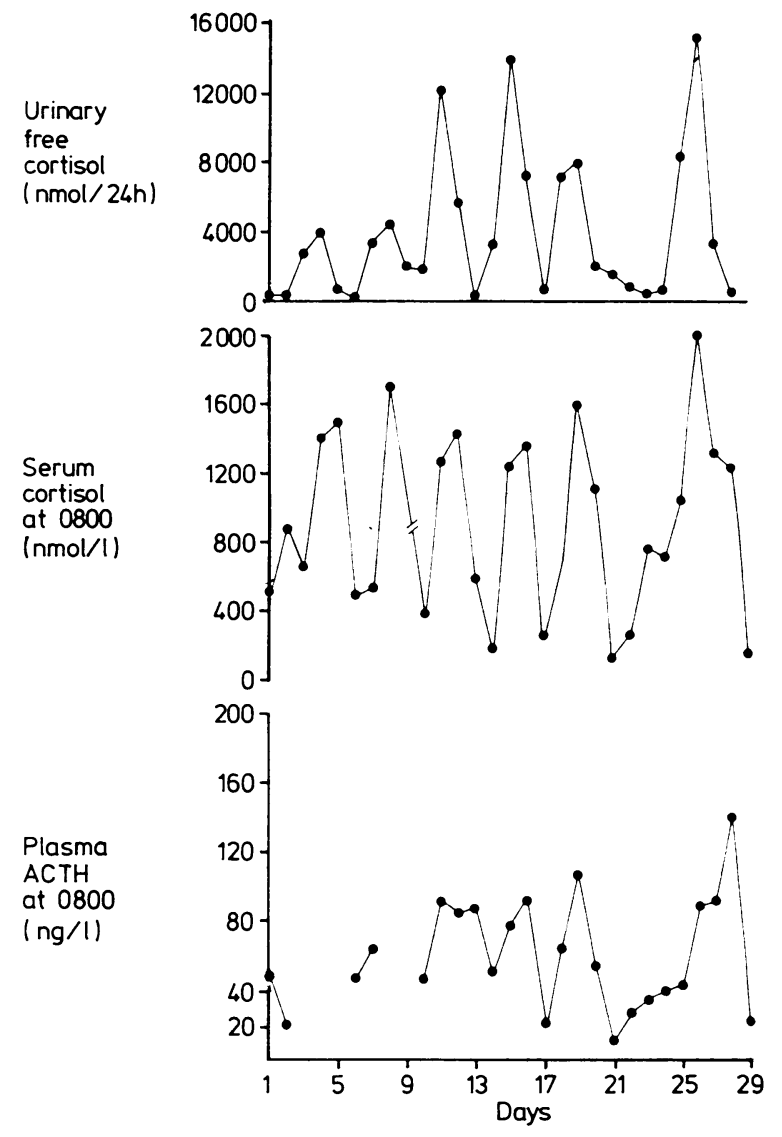

FIG 1-Case 1. 24 Hour urinary cortisol excretion and serum cortisol concentrations and plasma adrenocorticotrophic hormone (ACTH) values at 0800 over 29 days.

syndrome at another hospital. At that time she appeared Cushingoid with a bloated face and livid striae; urinary 17-ketosteroids were slightly raised at $13.1 \mathrm{mg} / 24 \mathrm{~h}$. Some weeks later, however, her appearance and urinary steroid excretion were normal and no further investigations were performed. About every two years over the next 16 years she had periods of illness lasting for two to three months during which she was emotionally unstable and 


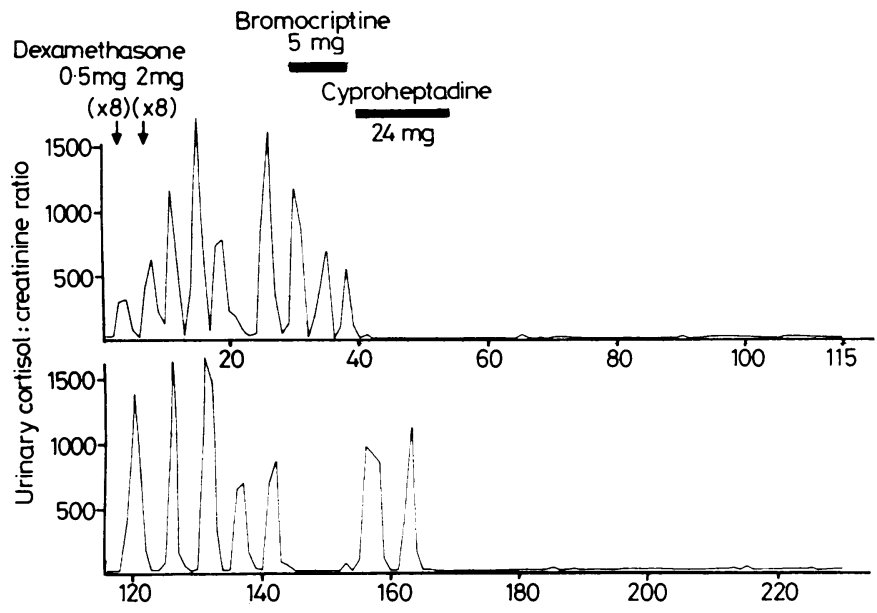

FIG 2-Case 1. Urinary cortisol:creatine ratios over 230 days. For days 1 to 79 the ratio is based on 24 hour urine collections; subsequently ratio is based on early morning specimen of urine. The upper limit of normal is 50 . tadine plus bromocriptine ( $10 \mathrm{mg}$ daily) interrupted the cyclical pattern or changed the amplitude of the peaks of cortisol production.

Transsphenoidal surgery was unsuccessful and bilateral adrenalectomy was therefore performed. The glands weighed $16 \mathrm{~g}$ and the histological features were of diffuse adrenal hyperplasia. The patient has been well since the operation.

CASE 3

A 39 year old woman presented to another hospital in 1981 with palpitations and chest pain. Supraventricular tachycardia was diagnosed and treated. Cushing's syndrome was suspected. On admission to our unit plethora, obesity, moon face, facial hirsutism, livid striae, and skin bruising were noted. Blood pressure was $150 / 100 \mathrm{~mm} \mathrm{Hg}$. There was loss of diurnal variation of serum cortisol. Dexamethasone only partially suppressed the 0800 serum cortisol concentration. At transsphenoidal hypophysectomy in August 1981 a basophil adenoma was removed.

After operation the patient was slow to lose her Cushingoid features. Urinary free cortisol and serum cortisol were variable over 10 months with some values in the normal range. Low dose dexamethasone suppression tests, performed twice after the operation, showed variable results (see
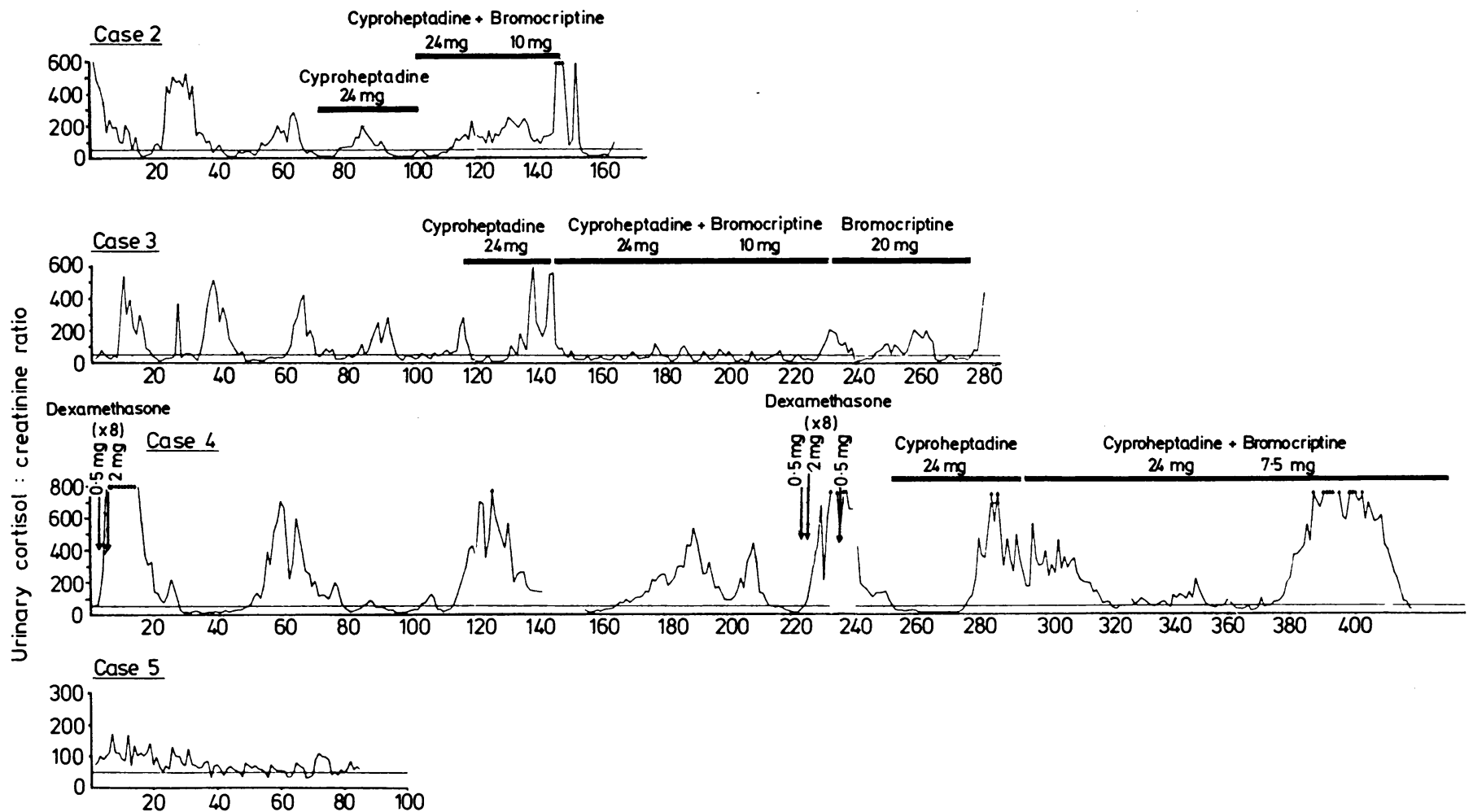

FIG 3-Urinary cortisol:creatinine ratios over extended periods in four patients with cyclical Cushing's syndrome. In case $4 \uparrow \uparrow \uparrow$ indicates values $>800$. Note that individual scales differ.

nervous. Two years before admission to our unit she noted considerable weight gain and facial swelling. Since then she had had regular episodes of weight gain, nervousness, and inability to cope. These episodes tended to occur premenstrually and her facial appearance fluctuated.

On examination on admission to this unit she had mild facial hirsutism and non-livid striae of the thighs. Blood pressure was $130 / 80 \mathrm{~mm} \mathrm{Hg}$. The results of two low dose and one high dose dexamethasone suppression tests (see table) showed considerable variation in baseline serum and urinary cortisol values. Mean plasma adrenocorticotrophic hormone was $26 \mathrm{ng} / 1$ during 24 hours when the serum cortisol was high. Computed tomography showed an enlarged pituitary gland with a convex upper border but no definite evidence of an adenoma. A chest radiograph was normal.

Sequential early morning specimens of urine were collected and analysed for free cortisol:creatinine ratio.

Fig 3 shows the cyclical pattern of secretion with normal values at the troughs. Neither cyproheptadine ( $8 \mathrm{mg}$ three times daily) nor cyprohep- table). Early morning urine specimens were collected and these showed a clear cvclical pattern of cortisol secretion (fig 3). Cyproheptadine alone was without effect. Cypropheptadine and bromocriptine reduced urinary cortisol:creatinine ratios, but cyproheptadine had to be withdrawn because of weight gain and somnolence. On bromocriptine alone urinary cortisol: creatinine ratios again rose. Bilateral adrenalectomy was performed with removal of hyperplastic glands (combined weight $21.5 \mathrm{~g}$ ). Postoperative recovery has been good.

CASE 4

This boy was originally referred at the age of 4 with sudden unexplained weight gain. Height and weight were on the 10th and 50th percentile respectively. Over the following year his mother noticed at least three episodes of facial and abdominal swelling, each lasting a few weeks and 
unaccompanied by other symptoms. At the age of $51 / 2$ he was admitted for investigation of weight gain $(2.5 \mathrm{~kg}$ in 10 days), hypertension $(140 / 70 \mathrm{~mm}$ $\mathrm{Hg}$ ), and glycosuria. At that time the serum cortisol concentration was 520 $\mathrm{nmol} / \mathrm{l}$ at 0900 and $599 \mathrm{nmol} / 1$ at 2300 . Twelve days later the 0900 cortisol value was $178 \mathrm{nmol} / 1$ and the evening value $186 \mathrm{nmol} / 1$. Three months after admission he had a left atrial myxoma removed. Over the next five years intermittent facial and abdominal swelling was recognised by his mother and he sometimes had mildly raised blood pressure. In view of the benign nature of his symptoms and his continued normal growth along the 10th percentile no extensive investigations were undertaken.

During this time variable urinary cortisol values of 641,146 , and 88 $\mathrm{nmol} / 24 \mathrm{~h}$ were noted. However, at the age of 10 his growth suddenly slowed and there was radiological evidence of reduced bone density of the wrist, lumbar vertebrae, and femora. At 12 years height had fallen to below the third percentile. During a low and high dose dexamethasone suppression test the urinary free cortisol and serum cortisol rose (see table). Fig 3 shows the results of early morning urinary cortisol:creatinine ratios. When urinary excretion of cortisol was high the plasma adrenocorticotrophic hormone values were higher than on the days when urinary free cortisol was low. Over the next seven months three peaks in cortisol excretion were identified, unrelated to any treatment, at days 61,134 , and 189. After three completed cycles and seven days of normal results a further dexamethasone suppression test increased serum and urinary cortisol values at a time slightly earlier than expected (fig 3). Cyproheptadine and bromocriptine failed to change the pattern of urinary cortisol secretion. Computerised tomography suggested a filling defect in the pituitary and a basophil adenoma was removed.

\section{CASE 5}

A 34 year old woman presented with mild bruising and plethora. Basal urinary free cortisol concentrations were raised and suppressed incompletely during low dose dexamethasone therapy. After the high dose test urinary free cortisol fell to $64 \mathrm{nmol} / 24 \mathrm{~h}$. Three months later repeat tests gave similar results (see table). When serum cortisol values were raised plasma adrenocorticotrophin values varied from 20 to $100 \mathrm{ng} / 1$. A conservative policy was adopted with frequent outpatient reviews. Daily early morning specimens of urine for a cortisol:creatinine ratio collected for three months (fig 3) showed a clear cyclical pattern with the cortisol secretion returning to normal several times. Subsequently she developed slight muscle weakness and her face became more plethoric. A computed tomogram of the pituitary suggested the presence of a microadenoma, which was subsequently confirmed surgically.

\section{Discussion}

The criteria for diagnosing cyclical Cushing's syndrome have been satisfied in only a few previous cases. Does this mean that the disease is extremely rare and that our recent rapid accumulation of cases is a coincidence? This may be so, but there have been several reports of patients with fluctuating urine or plasma cortisol values in whom repeated collections of specimens have not been obtained to establish or disprove a cyclical phenomenon. ${ }^{1+24}$ Several other workers have reported a paradoxical response to dexamethasone ${ }^{25 \cdot 27}$ but have made no attempt to establish whether there was a cyclical phenomenon. Brown et al did, however, perform prolonged studies after such a paradoxical response and established a diagnosis of cyclical Cushing's disease. ${ }^{2}$ They also established that the rise in cortisol values during dexamethasone therapy was not secondary to the dexamethasone; it merely coincided with administration of the drug. They concluded that any anomalous responses to dexamethasone should be verified by repeat testing and cyclical Cushing's considered as a differential diagnosis. Two of our five patients had a paradoxical response to dexamethasone. This was noted several times in case 1 (postoperative paradoxical response results not included) and case 4 . However, we have not maintained dexamethasone treatment over an extended period to see whether spontaneous independent fluctuations of cortisol still occurred and so cannot state definitely that dexamethasone caused the rise in cortisol concentration. Nevertheless, there remains the possibility that in some patients dexamethasone has an effect on the hypothalamicpituitary axis, causing release of adrenocorticotrophic hormone.

Why do some patients with Cushing's syndrome have cycles in their production of cortisol? Halberg has shown cycles of steroid production in normal people, ${ }^{28}$ so we might simply be observing an exaggeration of the normal cyclical variation in a subgroup of patients.

Our experience over two years suggests that cyclical Cushing's syndrome is not rare. Although the clinical condition of five of our 14 patients with Cushing's syndrome did not allow us to observe them untreated over an extended period, two did show considerable variation in cortisol production over short periods, though no cycles were established. Our experience suggests that the cyclical form of the disease should be more actively sought. The following are worthwhile clinical clues: variable cortisol values, paradoxical or inconsistent responses to dexamethasone, and a variable clinical syndrome such as that seen in cases 1,2, and 4. Patients slow to lose clinical features of hypercortisolism should also be screened after transsphenoidal surgery (case 3 ).

Two of our patients had long periods of normal health or normal cortisol production, which raises the possibility that we may miss the diagnosis of Cushing's syndrome more often than we think. Of interest are reports that at necropsy $20 \%$ to $30 \%$ of people have a pituitary adenoma ${ }^{29}{ }^{30}$ and $27 \%$ of these are basophil in type. ${ }^{29}$ The long periods of normal cortisol production also make it difficult either to exclude or to diagnose cortisol overproduction because confirmation demands prolonged daily collections of urine for cortisol estimation. Where the possibility has to be vigorously pursued the mailed early morning specimen of urine is invaluable.

It has been suggested that the dopamine agonist bromocriptine ${ }^{21.33}$ and the serotonin antagonist cyproheptadine may be helpful in the management of some patients with Cushing's disease. ${ }^{34-36}$ Our studies with bromocriptine and cyproheptadine either alone or in combination do not suggest that the dopaminergic and serotoninergic systems are concerned in regulating the cycles. Only the third patient showed a response to the combination of cyproheptadine and bromocriptine but we could not attempt to verify this because of side effects.

In summary, we suggest that cyclical Cushing's syndrome is more common than suspected. The possibility of cyclical steroidogenesis should caution against attributing falls in cortisol values to drugs in single case reports or non-randomised trials. Furthermore, a high index of suspicion for the condition is required in patients with symptoms or signs of Cushing's syndrome but with normal or variable cortisol values, in patients with fluctuating cortisol values, and in those with the so called paradoxical response to dexamethasone.

We thank Mr T Fannin, Mr D S Gordon, and Mr T L Kennedy for performing the operations described, the nursing staff of the metabolic unit, Royal Victoria Hospital, and Musgrave Ward, Royal Belfast Hospital for Sick Children, for their skill, Miss C Gilmartin for preparing the illustrations, and Mrs M Loughran for preparing the manuscript.

\section{References}

1 Bailey RE. Periodic hormonogenesis-a new phenomenon. Periodicity in function of a hormoneproducing tumour in man. $\mathcal{J}$ Clin Endocrinol 1971;32:317-27.

2 prown RD, Van Loon GR, Orth DN, Liddle GW. Cushing's disease with periodic hormonogenesis: one explanation for paradoxical response to dexamethasone. $\mathcal{f}$ Clin Endocrinol Metab genesis: one explan $1973 ; 36: 445-51$.

3 Birke G, Diczfalusy E, Plantin L. Fluctuations in the excretion of adrenocortical steroids in a case of Cushing's syndrome. I Clin Endocrinol 1956;16:286-90.

4 Chajek T, Romanoff H. Cushing's syndrome with cyclical edema and periodic secretion of corticosteroids. Arch Intern Med 1976;136:441-43.

5 Liberman B, Waichenberg BL, Tambascia MA, Mesquita CH. Periodic remission in Cushing's disease with paradoxical dexamethasone response: an expression of periodic hormonogenesis. J Clin Endocrinol Metab 1976;43:913-8.

6 Oates TW, McCourt JP, Friedman WA, Agee OF, Rhoton AL, Thomas WC. Cushing's disease with cyclical hormonogenesis and diabetes insipidus. Neurosurgery 1979;5:598-603.

7 Cook DM, Kendall JW, Jordan R. Cushing's syndrome. Current concepts of diagnosis and treatment. West f Med 1980;132:111-22.

8 Schteingart DE, McKenzie AK. Twelve-hour cycles of adrenocorticotrophin and cortisol secretion in Cushing's disease. $\mathcal{F}$ Clin Endocrinol Metab 1980;51:1195-8.

9 Jordan RM, Ramos-Gabatin A, Kendall JW, Gaudette D, Walls RC. Dynamics of adrenocorticotropin (ACTH) secretion in cyclic Cushing's syndrome: evidence for more than one abnormal tropin (ACTH) secretion in cyclic Cushing's syndrome: e
ACTH biorhythm. $\mathcal{F}$ Clin Endocrinol Metab 1982;55:531-7.

10 CCTH biorhythm. F Clin Endocrnol Metab 1982;55:531-7.

11 Riad-Fammy D, Read GF, Gaskell SJ, Dyas J, Hindawi R. A simple direct radioimmunoassay for plasma cortisol featuring a ${ }^{125}$ I radioligand and a solid phase separation technique. Clin Chem
(n)

12 Cook JGM. Factors influencing the assay of creatinine. Ann Clin Biochem 1975;12:219-32.

13 Atkinson AB, Chestnutt A, Crothers E, et al. Cyclical Cushing's disease- two distinct rhythms in a patient with a basophil adenoma. $\mathcal{O}$ Clin Endocrinol Metab 1985;60:328-32. 
14 Scott RS, Espiner EA, Donald RA. Intermittent Cushing's disease with spontaneous remission. Clin Endocrinol 1979;11:561-6.

15 Cushing $\mathrm{H}$. The basophil adenomas of the pituitary body and their clinical manifestations pituitary basophilism). Bulletin of the fohns Hopkins Hospital 1932;50:137-95.

16 Anderson E, Haymaker W. Cushing's syndrome, 7 Ment Nerv Dis 1944:99-511-20.

17 Pasqualini RQ, Gurevich N. Spontaneous remission in a case of Cushing's syndrome. $7 \mathrm{Clin}$ Endocrinol Metab 1956:16:406-11.

18 Bassöe HH, Emberland R, Stöa KF. Fluctuating steroid excretion in Cushing's syndrome. Acta Endocrinol Copenh 1958;28:163-8.

19 Aber CP, Cheetham HD. Cyclical Cushing's syndrome. Br Med 7 1961;: 336-8.

20 Ovlisen B, Andersen HJ. Spontaneous remission in a case of Cushing's svndrome presumably due lisen B, Andersen H. Spontaneous remission in a

21 Ruder HJ, Loriaux DL, Lipsett MB. Severe osteopenia in young adults associated with Cushing's syndrome due to micronodular hyperplasia. $\mathcal{F}$ Clin Endocrinol Metab 1974;39:1138-47.

22 Blau N, Miller WE, Miller ER, Cervi-Skinner SJ. Spontaneous remission of Cushing's syndrome in a patient with an adrenal adenoma. $f$ Clin Endocrinol Metab 1975;40:659-63.

23 Green JRB, Van't Hoff $W$. Cushing's syndrome with fluctuation due to adrenal adenoma. $\mathcal{F}$ Clin Endocrinol Metab 1975;41:235-40.

24 Bochner F, Burke CJ, Lloyd HM, Nurnberg BI. Intermittent Cushing's disease. Am J Med 1979;69:507-10.

25 James VHT, Landon J, Wynn V. Oral and intravenous suppression tests in the diagnosis of Cushing's syndrome. I Endocrinol 1965;33:515-24.

26 Brooks RV, Jeffcoate SL, London DR, Prunty FTG, Smith PM. Intermittent Cushing's syndrome with anomalous response to dexamethasone. $\mathcal{F}$ Endocrinol 1966;36:53-61.
27 French FS, Macfie JA, Baggett B, Williams TF, Van Wyk JJ. Cushing's syndrome with a paradoxical response to dexamethasone. Am f Med 1969;47:619-24.

28 Halberg F, Engeli $M$, Hamburger $C$. The 17-ketosteroid excretion of a healthy man on weekdays and weekends. Exp Med Surg 1965;23:61-69.

29 Costello RT. Subclinical adenoma of the pituitary gland. Am f Pathol 1936;12:205-15.

30 Burrow GN, Wortzman G, Rewcastle NB, Holgate RC, Kovacs K. Microadenomas of the pituitary and abnormal sellar tomograms in an unselected autopsy series. $N$ Engl $\mathrm{f} \mathrm{Med}$ 1981;304:156-8.

31 Spark RF, Dickstein G. Bromocriptine and endocrine disorders. Ann Intern Med 1979;90:949-56

32 Lamberts SWJ. Klijn JGM. De Quijada M et al The mechanism of the suppressive action of bromocriptine on adrenocorticotropin secretion in patients with Cushing's disease and Nelson's undrome. F Clin Endocrinol Metab 1980:51:307-11.

33 Kennedy AL, Sheridan B, Montgomery DAD. ACTH and cortisol response to bromocriptine, and results of long-tern therapy in Cushing's disease. Acta Endocrinol Copenh 1978;89:461-8. 34 Krieger DT, Amorosa L, Linick F. Cyproheptadine-induced remission of Cushing's disease. NEngl J Med 1975;293:893-6.

35 Grant DB, Atherden SM. Cushing's disease presenting with growth failure: clinical remission during cyproheptadine therapy. Arch Dis Child 1979;54:466-8

36 Hsu T, Gann DS, Tsan K, Russell RP. Cyproheptadine in the control of Cushing's disease. Fohns Hopkins Medical Journal 1981;149:77-83.

\title{
Long term effects of cyclophosphamide on testicular function
}

\author{
A R WATSON, C P RANCE, J BAIN
}

\begin{abstract}
Thirty men treated in childhood with cyclophosphamide for a mean of 280 days were assessed at a mean of 12.8 years after treatment for hormone concentrations and spermatogenesis. Four were azoospermic, nine oligospermic, and 17 normospermic. There was a significant inverse correlation of sperm density with cyclophosphamide dosage and duration of treatment. After a further mean follow up of $7 \cdot 2$ years three patients who were previously oligospermic and one who was azoospermic had normal sperm counts. All patients had normal sexual characteristics and libido. Serum androgen and prolactin concentrations did not differ significantly between patients and controls. Raised basal and stimulated follicle stimulating hormone concentrations were in keeping with impaired spermatogenesis. All patients had significantly raised luteinising hormone responses on stimulation with luteinising hormone releasing hormone.

The results suggest compensated Leydig cell failure, and patients with this condition require long term evaluation of testicular function. Potential recovery of spermatogenesis with time requires appropriate counselling and contraceptive advice.
\end{abstract}

\section{Introduction}

Early controlled clinical trials showed that cyclophosphamide effectively maintained remission of the childhood nephrotic

\footnotetext{
Division of Nephrology, The Hospital for Sick Children, Toronto, Ontario, Canada M5G 1 X8

A R WATSON, MB, MRCP, consultant nephrologist

C P RANCE, MD, FRCP, consultant nephrologist

Division of Endocrinology and Metabolism, Mount Sinai Hospital, Toronto, Ontario, Canada

J BAIN, MD, FRCP, consultant endocrinologist
}

Correspondence to: Dr Watson, Department of Paediatrics, City Hospital, Nottingham NG5 1PB. syndrome in patients who were sensitive to steroids and had frequent relapses. ${ }^{1-3}$ Until 1972 the gonadal toxicity of this drug was not appreciated. ${ }^{4}$ Subsequent reports, however, have suggested a significant inverse correlation of sperm density with cyclophosphamide dosage..$^{6-8}$

In adult patients rendered azoospermic by cyclophosphamide recovery of spermatogenesis has been documented for up to four years after treatment, a finding that has obvious implications for fertility counselling and contraceptive advice. ${ }^{9}$ Little information is available on the long term outcome with respect to recovery of spermatogenesis in patients who were treated with large doses of cyclophosphamide during childhood. In addition, as most childhood patients have been studied either prepubertally or in early adulthood reports on their testicular hormone and gonadotrophin concentrations have been confusing. ${ }^{10-12}$

This study correlates gonadal effects with cyclophosphamide dosage in a large, single centre study and documents any recovery of spermatogenesis in patients who were tested and reported on previously. ${ }^{8}$ In addition, the responses of the sex hormones of such patients were determined.

\section{Patients and methods}

Men who were aged 17 or older in 1983 and had been treated with cyclophosphamide for childhood nephrotic syndrome at The Hospital for Sick Children, Toronto, between 1967 and 1976 were considered eligible for this study. Of 70 such patients, 28 were unavailable, two had died in motor vehicle accidents, seven refused to participate, and 33 were included in the study. Three patients were subsequently excluded from analysis owing to chronic renal failure (two patients) or a single testis (one patient).

In the 30 remaining patients histological examination before treatment with cyclophosphamide showed minimal lesions in 27 and focal segmental glomerulosclerosis in three. They had been treated with cyclophosphamide at a dose of $2-3 \mathrm{mg} / \mathrm{kg}$ body weight/day for a mean of 280 days (range 42-556). Thirteen patients had received treatment for a year or longer. Two had undergone two courses of treatment roughly two years apart, and the sum of the two courses was used to calculate the dose of cyclophosphamide.

When reviewed in 1983 all 30 patients were well and free of oedema. The three who had focal segmental glomerulosclerosis continued to have proteinuria with no clinical evidence of nephrosis or serious renal impairment. Two of the six patients with minimal lesions who relapsed after 